\title{
Influência da satisfação acadêmica na comunicação de enfermeiros
}

\author{
Influence of academic satisfaction on the communication skills of nurses \\ Influencia de la satisfacción académica en la comunicación de enfermeros
}

\begin{abstract}
RESUMO
Objetivos: Correlacionar a satisfação acadêmica com a comunicação interpessoal do enfermeiro; associar as características sociodemográficas e profissionais com a comunicação interpessoal do enfermeiro. Método: Estudo analítico transversal. Utilizou-se a Escala de Satisfação com a Experiência Acadêmica de Estudantes do Ensino Superior; a Escala de Competência em Comunicação Interpessoal e a Escala sobre Profissionalismo e Competência em Comunicação Interpessoal Enfermeiro e Paciente. Resultados: Participaram 397 enfermeiros. Enfermeiros com menor tempo de formação, mais jovens e católicos, demonstraram mais disponibilidade nas relações; os que estudaram em instituições públicas mostraram mais habilidade para expressar sentimentos; aqueles que não tinham formação prévia na área de enfermagem foram mais capazes de demonstrar compreensão e perceber o que as pessoas sentem; tanto aqueles que se declararam católicos quanto sem religião referiram ter mais sinceridade nas relações enfermeiro-paciente. Conclusões: Quanto mais satisfeitos com a experiência acadêmica, melhor é a comunicação interpessoal do enfermeiro.

Descritores: Relações Interpessoais; Profissionalismo; Educação em Enfermagem.
\end{abstract}

\section{ABSTRACT}

Objectives: To correlate academic satisfaction with nurses' interpersonal communication skills; to associate sociodemographic and professional characteristics with nurses' interpersonal communication skills. Method: This is a cross-sectional analytical study. We used the Scale of Satisfaction with the Academic Experience of Students of Higher Education, the Competence Scale in Interpersonal Communication and the Scale about Professionalism and Competence in Interpersonal Communication Nurse and Patient. Results: 397 nurses participated in this study. Less experienced, younger, catholic nurses showed more openness to relationships; those who studied in public institutions showed more ability to express their feelings; those who did not have prior training in the nursing area were better able to demonstrate understanding and notice what people feel; those who declared themselves Catholic and non-religious showed more sincerity in the nurse-patient relationship. Conclusions: The more satisfied with their academic experience, the better the nurses' interpersonal communication.

Descriptors: Interpersonal Relations; Professionalism; Nursing Education.

\section{RESUMEN}

Objetivos: Correlacionar la satisfacción académica con la comunicación interpersonal del enfermero; Asociar las características sociodemográficas y profesionales con la comunicación interpersonal del enfermero. Método: Estudio analítico transversal. Se utilizó la Escala de Satisfacción con la Experiencia Académica de Estudiantes de Enseñanza Superior, la Escala de Competencia en Comunicación Interpersonal y la Escala sobre Profesionalismo y Competencia en Comunicación Interpersonal Enfermero y Paciente. Resultados: Participaron 397 enfermeros. Los enfermeros con menor tiempo de formación, más jóvenes y católicos demuestran más disponibilidad en las relaciones; Los que estudiaron en instituciones públicas mostraron más habilidad para expresar sentimientos; Los que no tenían formación previa en el área de enfermería fueron más capaces de demostrar comprensión y percibir lo que las personas sienten; Tanto aquellos que se declararon católicos así como sin religión referirán tener más sinceridad en las relaciones enfermero-paciente. Conclusiones: Cuanto más satisfechos con la experiencia académica, mejor es la comunicación interpersonal del enfermero.

Descriptores: Relaciones Interpersonales; Profesionalismo; Educación en Enfermería.
Francine da Costa Alves ${ }^{1}$

$\underline{0000-0002-3768-9476}$

Ana Claudia Giesbrecht Puggina 2 (iD) 0000-0001-8095-6560

1 Universidade de Taubaté.

2 Faculdade de Medicina de Jundiaí.

Autor correspondente:

Francine da Costa Alves

E-mail: enffran tte@hotmail.com

\section{Como citar este artigo:}

Alves FC, Puggina ACG. Influência Da satisfação acadêmica na comunicação do enfermeiro. Revista de Enfermagem do Centro-Oeste Mineiro. 2021;11:e4000. [Access___ Available in:__. DOI: http://doi.org/10.19175/recom.v11i0.40 $\underline{00}$ 


\section{INTRODUÇÃO}

As instituições de ensino superior desempenham importante papel na formação dos acadêmicos e devem, em todo o processo de formação, disponibilizar recursos e proporcionar experiências construtivas para o desenvolvimento profissional $^{(1)}$. O enfermeiro, durante a fase de formação, adquire conhecimentos teóricos e práticos $^{(2)}$ com o objetivo de preparar-se para o exercício de sua profissão e adquirir habilidades gerais de atenção à saúde, anamnese e exame físico, tomada de decisão, liderança, administração, gerenciamento, educação permanente e comunicação ${ }^{(3)}$.

Espera-se também que, ainda durante a formação, esse aluno vivencie situações da prática do ser enfermeiro com exercício da autonomia, responsabilidade e atenção nas relações profissional-paciente, para que futuramente se sintam confiantes, seguros e capacitados para prestar cuidados de enfermagem, considerando o paciente na sua totalidade e complexidade ${ }^{(4)}$. Evidências apresentadas, em um estudo qualitativo, sinalizam para a necessidade de uma organização à formação acadêmica do enfermeiro, que desenvolva pessoas dinâmicas, interativas, reflexivas, críticas, com conhecimentos e habilidades humanizadas e que agreguem valores sociais à sua atuação, podendo colocar-se em outras posições no mercado de trabalho(5).

No exercício da profissão, o enfermeiro desenvolve várias ações voltadas ao cuidar, gerenciar, educar e pesquisar ${ }^{(6)}$, em todas essas ações, há inúmeros conhecimentos técnicos que devem ser aprendidos, sendo assim, desenvolver habilidades em comunicação pode facilitar muito essas ações, dar fluidez às interações com o paciente, família ou equipe e permitir que o enfermeiro compreenda o seu contexto de uma forma mais holística.

Verifica-se que as expectativas dos alunos, em relação ao aprendizado das questões relacionadas à comunicação, sentimentos, comportamentos, medos e humanização, geralmente, não são atendidas. Essas questões surgem na formação de maneira fragmentada e com um distanciamento entre o que é ensinado e o que é vivenciado ${ }^{(7)}$. Os enfermeiros necessitam de melhor preparo, conhecimento e treinamento, para utilizar a comunicação com eficiência e eficácia, o que poderia ser adquirido não somente em disciplinas específicas de comunicação e relacionamento profissional-paciente, como também na reflexão adequada sobre essas habilidades nas atividades práticas do curso $^{(8)}$.

Destaca-se a importante relação entre 0 domínio do conhecimento e habilidades em comunicação do enfermeiro, visto que toda comunicação tem obrigatoriamente duas partes: o conteúdo (fato, informação ou conhecimento técnico-prático que depende da formação) e o sentimento ou intenção (o que o profissional quer comunicar e como se sente a respeito desse fato, informação ou conhecimento) $)^{(9)}$. Sem o conhecimento do ser enfermeiro na sua totalidade, as interações com o paciente podem ficar superficiais e sem metas terapêuticas.

Na comunicação, processo de transmitir e receber mensagens, por meio de símbolos (estímulos que possuem uma única decodificação possível) ou sinais (estímulos que possuem mais de um significado), a mensagem é um elemento tão importante quanto o emissor, receptor, os canais pelos quais ela ocorre (maneira) ou efeitos que são produzidos nos indivíduos (resposta) ${ }^{(9)}$.

Fragilidades de aprendizagem, durante a formação, certamente, podem impactar na atuação comunicativa do futuro profissional. Para realizar adequadamente a anamnese e o exame físico de um paciente, por exemplo, o enfermeiro precisa tanto do conhecimento técnico quanto das habilidades em comunicação.

Considerando mensagem, conhecimento técnico-prático ou metaterapêutica, como partes essenciais do processo comunicativo do enfermeiro, comunicação e conhecimento tornam-se elementos indissociáveis e dependentes. Portanto propõe-se neste estudo conhecer a influência da satisfação acadêmica nas habilidades em comunicação do enfermeiro.

Entende-se que satisfação é algo variável, porque as pessoas possuem necessidades diferentes umas das outras em determinados momentos e circunstâncias ${ }^{(10)}$. Refere-se a um processo dinâmico e recíproco em que, por um lado, os estudantes modificam o ambiente, a partir de suas percepções e, por outro, o ambiente gera impacto nos estudantes em decorrência das experiências possibilitadas ${ }^{(10)}$. Entretanto a satisfação com a experiência acadêmica pode refletir mais diretamente se um resultado esperado pelo indivíduo foi alcançado e está sendo essencial na sua atuação(2).

Conhecer as satisfações ou insatisfações dos estudantes quanto à formação pode contribuir para o entendimento do impacto do ensino superior na atuação do profissional, já que o 
desencontro entre as expectativas dos estudantes, o que a instituição oferece e o que a realidade profissional necessita pode gerar baixo desempenho e insegurança ${ }^{(11)}$. Não há dúvidas de que a graduação é um período valioso, para que o processo de formação profissional aconteça, pois é nele que se adquirem conhecimentos e habilidades fundamentais da profissão; no entanto é importante destacar que a identidade profissional certamente se desenvolve, ao longo da vida, com estudo, experiências e com reflexões sobre a forma de agir, pensar e se relacionar ${ }^{(12)}$. Dessa forma, este artigo objetivou correlacionar a satisfação acadêmica com a comunicação interpessoal do enfermeiro e associar as características sociodemográficas e profissionais com a comunicação interpessoal do enfermeiro.

\section{MÉTODO}

Estudo analítico transversal. As variáveis dependentes foram a competência em comunicação interpessoal e a autoavaliação sobre profissionalismo. As independentes foram a satisfação acadêmica, características sociodemográficas e profissionais.

Foram incluídos na pesquisa enfermeiros assistenciais que atuam ou atuaram na área assistencial com adultos e que possuíam perfil na rede social Facebook ${ }^{\circledR}$. Foram excluídos do estudo enfermeiros com atuação exclusiva, nas áreas de pediatria e neonatologia, ou que nunca atuaram na área assistencial, porque uma das escalas contempla o exame físico em adultos.

A coleta de dados ocorreu de junho a novembro de 2016, por meio de recrutamento direto e indireto. No recrutamento direto, os enfermeiros foram abordados no ambiente de trabalho e, após a explicação sobre a pesquisa, convidados a participar. Para os que aceitaram, foi solicitado o seu perfil na rede social Facebook ${ }^{\circledR}$ e, posteriormente, enviado um convite individual via mensagem no Facebook $^{\circledR}$ com link de acesso ao questionário.

No recrutamento indireto, enfermeiros membros de grupos públicos relacionados à enfermagem, da rede social Facebook ${ }^{\circledast}$, foram convidados a participar da pesquisa por meio de um link publicado na linha do tempo dos grupos. Enfermeiros com perfis pessoais vinculados aos pesquisadores foram convidados por mensagem privada a colaborar com a pesquisa.

Foi estimado um valor médio referente à população de interesse para este estudo por meio do Laboratório de Epidemiologia e Estatística do Instituto Dante Pazzanese de Cardiologia. Ao calcular a amostra dessa forma, supõe-se que a variável que contenha a resposta de interesse siga uma distribuição normal com desvio-padrão supostamente conhecido.

Considerando o desvio-padrão $( \pm 3,99)$ e média $(47,8)$ de estudo anterior da Escala de Autoavaliação sobre Profissionalismo e Competência em Comunicação Interpessoal entre Enfermeiros e Pacientes ${ }^{(13)}$ e o erro máximo da estimativa correspondente a $10 \%$ do desviopadrão $(E M E=0,39)$ e fixando nível de significância de $5 \%$, o valor médio estimado para a aplicação desse instrumento foi de 384 .

O cálculo amostral refere-se ao tamanho mínimo necessário para a aplicação do instrumento. O período de coleta de dados foi de junho a novembro de 2016, totalizando cinco meses. Não foi possível mensurar o número de pessoas que tiveram acesso ao link da pesquisa; diariamente os grupos públicos da rede social Facebook mudam o número de membros.

Todos os participantes responderam aos questionários da mesma maneira pelo link de acesso ao questionário construído no Google forms ${ }^{\circledR}$. Ao clicar no link, a primeira tela era do Termo de Consentimento Livre e Esclarecido; o consentimento do participante do estudo foi obtido, por meio de aceite eletrônico, ou seja, o participante tinha que clicar em "aceito participar da pesquisa" para prosseguir. Os que clicavam em "não aceito participar da pesquisa" eram direcionados a uma página de agradecimento. Nessa etapa, doze pessoas clicaram em não participar da pesquisa.

Para confirmar que o participante era enfermeiro e prevenir duplicidades, foi solicitada a identificação com o nome completo e número de inscrição no Conselho Regional de Enfermagem (Coren), logo depois, conferida nos sites dos conselhos regionais essa informação. Sendo assim, os participantes que não eram enfermeiros e que não atendiam aos critérios de inclusão, bem como profissionais que responderam mais de uma vez, foram excluídos da amostra.

Foram utilizados quatro questionários. O questionário de caracterização dos participantes continha variáveis sociodemográficas (idade, naturalidade, sexo, estado civil, cor autorreferida, religião, renda pessoal) e profissionais (escolaridade completa, tempo de formação, instituição de nível superior, participação em atividades acadêmicas, formação prévia em 
enfermagem, turno de trabalho e unidade de atuação).

A Escala de Satisfação com a Experiência Acadêmica de Estudantes do Ensino Superior (ESEA) continha 35 itens divididos em três dimensões (satisfação com o curso, oportunidade de desenvolvimento e satisfação com a instituição), mensuradas por respostas do tipo Likert de cinco pontos, que variaram de "nada satisfeito" a "totalmente satisfeito". O escore total da escala variou de 55 a 175, quanto maior o escore, maior a satisfação com a experiência acadêmica(11). $^{(11}$.

Foi também utilizada a Escala de Competência em Comunicação Interpessoal (ECCI), com 17 itens, composta por cinco domínios (autorrevelação, assertividade, manejo das interações, controle do ambiente, disponibilidade) com respostas do tipo Likert de cinco pontos, variando de "nunca" a "quase sempre". Os itens 8 e 17 possuíam código reverso e deviam ser recodificados antes da somatória do escore total. A variação do escore total da escala foi de 17 a 85, quanto mais alto o escore da $\mathrm{ECCl}$, maior a competência em comunicação interpessoal do indivíduo(14). A Escala de Autoavaliação sobre Profissionalismo e Competência em Comunicação Interpessoal entre Enfermeiros e Pacientes (EAPCI) continha 11 itens, composta por quatro fatores, com respostas do tipo Likert de cinco pontos, variando de "nunca" a "sempre". O escore total variou de 11 a 44 e quanto maior o escore, maior a autoavaliação sobre profissionalismo e competência em comunicação com o paciente durante o exame físico ${ }^{(13)}$.

Os dados foram analisados pelo programa estatístico R Core Team $2016{ }^{\circledR}$ (GPL, 2016, Viena: Áustria). Foi realizada análise descritiva (frequência absoluta, relativa, medidas de posição e desviopadrão), inferencial, análise de regressão normal múltipla $(E C C I)$ e análise de regressão binomial negativa (EAPCI).

Para a comparação das variáveis quantitativas com os escores do instrumento, foi utilizado o Teste de Correlação de Spearman; na associação das variáveis categóricas com os escores, foram utilizados o Teste de Kruskal-Wallis e o Teste de Mann-Whitney. O nível de significância adotado nos testes foi de 0,05.

Quando o teste de associação foi significativo, foram analisados os $p$-valores nas comparações dois a dois pelo critério de Bonferroni e os grupos diferentes entre si foram aqueles com $\mathrm{p}$-valores menores que o geral.

O desenvolvimento do estudo atendeu as normas nacionais e internacionais de ética em pesquisa envolvendo seres humanos e foi aprovado pelo Comitê de Ética e Pesquisa sob o número de Parecer 1.562.760 e Certificado de Apresentação para Apreciação Ética (CAEE) N 53654216.0.0000.5506.

\section{RESULTADOS E DISCUSSÕES}

Este estudo contribuiu para enfatizar a importância de que conhecer a relação que existe entre a satisfação acadêmica, a comunicação e profissionalismo pode elucidar questões importantes que precisam ser revistas na formação do enfermeiro.

A amostra do estudo foi constituída de 397 enfermeiros com média de idade de 34,72 anos $( \pm 8,10)$, com tempo médio de formação de 6,84 anos $( \pm 5,15)$. A maioria era do sexo feminino, católicos e brancos, provenientes da região Sudeste. As maiores frequências foram de participantes casados e com até três salários mínimos de renda (Tabela 1).

A maioria foi de profissionais com especialização, formados em universidades privadas, sem formação prévia na área de enfermagem e participaram de eventos científicos durante a graduação. Foram mais frequentes enfermeiros com atuação em unidades de internação e em turno de trabalho integral (8 horas por dia) (Tabela 1).

Tabela 1 - Descrição das características dos participantes da pesquisa, Guarulhos, SP, 2017

\begin{tabular}{lccccc}
\hline Características & $\mathbf{N}^{\circ}$ & $\%$ & Características & $\mathbf{N}^{\circ}$ & $\%$ \\
\hline Sexo & & & Escolaridade & & \\
Feminino & 363 & 91,4 & Especialização & 212 & 53,4 \\
Masculino & 34 & 8,5 & Graduação & 168 & 42,3
\end{tabular}


Tabela 1 - Descrição das características dos participantes da pesquisa, Guarulhos, SP, 2017

\begin{tabular}{|c|c|c|c|c|c|}
\hline Características & $\mathrm{N}^{\circ}$ & $\%$ & Características & $\mathrm{N}^{\circ}$ & $\%$ \\
\hline Estado Civil & & & Mestrado & 17 & 4,2 \\
\hline Casado & 195 & 49,1 & Instituição de Formação & & \\
\hline Solteiro & 163 & 41,0 & Privada & 331 & 83,3 \\
\hline Divorciado & 28 & 7,0 & Publica & 66 & 16,6 \\
\hline Separado & 8 & 2,0 & Participação em & & \\
\hline Viúvo & 3 & 0,7 & Eventos Científicos & 136 & 34,2 \\
\hline Cor Autorreferida* & & & Não Participou & 103 & 25,9 \\
\hline Branca & 281 & 70,7 & Iniciação Científica & 98 & 24,6 \\
\hline Parda & 84 & 21,2 & Projetos de Extensão & 60 & 15,1 \\
\hline Preta & 25 & 6,3 & Formação Prévia na Área & & \\
\hline Amarela & 6 & 1,5 & Não & 218 & 54,9 \\
\hline Indígena & 1 & 0,2 & Técnico de Enfermagem & 119 & 29,9 \\
\hline Religião & & & Auxiliar de Enfermagem & 60 & 15,1 \\
\hline Católico & 216 & 54,4 & Turno de Trabalho & & \\
\hline Evangélico & 97 & 24,4 & Integral & 148 & 37,2 \\
\hline Espírita & 44 & 11,0 & $12 \times 36$ Noturno & 93 & 23,4 \\
\hline Testemunha de Jeová & 5 & 1,2 & Manhã & 66 & 16,6 \\
\hline Sem religião & 35 & 8,8 & 12×36 Diurno & 48 & 12,0 \\
\hline Renda (salário mínimo) & & & Vespertino & 42 & 10,5 \\
\hline 3 & 116 & 29,2 & & & \\
\hline 4 & 96 & 24,1 & Unidade de atuação & & \\
\hline 7 ou mais & 55 & 13,8 & Unidade de Internação & 165 & 41,5 \\
\hline 5 & 54 & 13,6 & Pronto Socorro & 78 & 19,6 \\
\hline 2 & 34 & 8,5 & UTI ou Semi-intensiva & 56 & 14,1 \\
\hline 6 & 25 & 6,3 & Ambulatório & 45 & 11,3 \\
\hline 1 & 17 & 4,2 & PSF/UBS & 26 & 6,5 \\
\hline Naturalidade & & & $\begin{array}{l}\text { Centro Cirúrgico e Centro } \\
\text { Obstétrico }\end{array}$ & 18 & 4,5 \\
\hline Sudeste & 337 & 84,8 & Maternidade & 9 & 2,2 \\
\hline Nordeste & 31 & 7,8 & & & \\
\hline Sul & 10 & 2,5 & & & \\
\hline Norte & 9 & 2,2 & & & \\
\hline Centro-Oeste & 9 & 2,2 & & & \\
\hline Outra & 1 & 0,2 & & & \\
\hline Total & 397 & 100 & Total & 397 & 100 \\
\hline
\end{tabular}

O escore médio total das respostas dos enfermeiros, em relação à ESEA, foi de 113,57 $( \pm 20,62)$. As maiores médias foram nos itens 9,5 e
13, e as menores médias, nos itens 6, 10, 23 (Figura $1)$.

Figura 1 - Descrição das maiores e menores médias dos itens respondidos na ESEA. Guarulhos, SP, Brasil, 2016. (N=397)

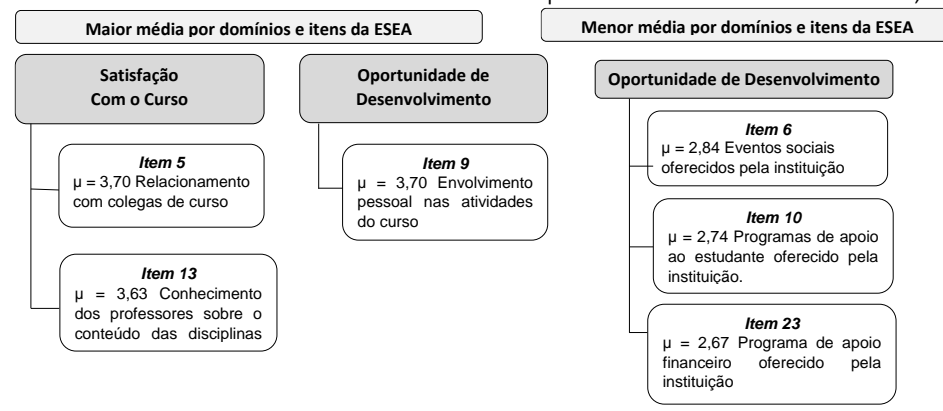

Fonte: Elaboração Própria (2020).

Legenda: ESEA: Escala de Satisfação com a Experiência Acadêmica de Estudantes do Ensino Superior. $\mu$ : média. 
O escore médio total das respostas, em relação à $\mathrm{ECCl}$, foi de $64,62( \pm 5,92)$ e, em relação à EAPCl, foi de 47,91 $( \pm 4,91)$. As maiores médias na $\mathrm{ECCl}$ foram nos itens 10, 11 e 14 , e as menores médias, nos itens 4, 8, 17. A EAPCl apresentou maiores médias nos itens 3,5 e 10 e menores médias nos itens 11, 1 e 7 (Figura 2).

Figura 2 - Descrição das maiores e menores médias dos itens respondidos na ECCl EAPCl. Guarulhos, SP, Brasil, 2016. (N=397)
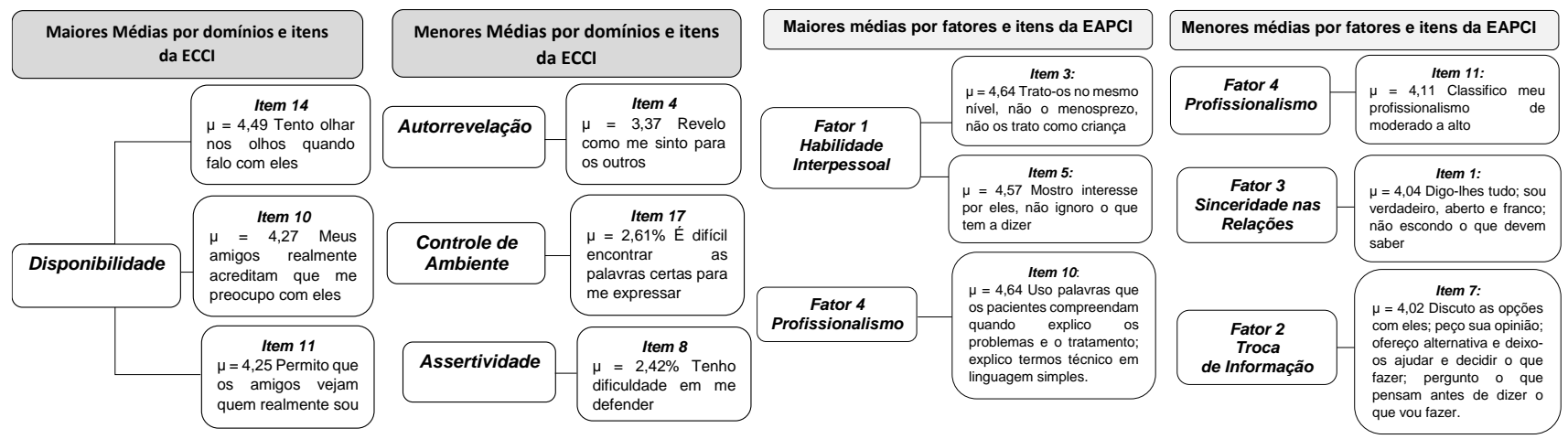

Fonte: Elaboração Própria (2020).

Legenda: ECCl: Escala de Competência em Comunicação Interpessoal. EAPCl: Escala de Autoavaliação sobre Profissionalismo e Competência em Comunicação Interpessoal entre Enfermeiros e Pacientes. $\mu$ : média.

A ESEA apresentou correlação positiva e significativa com a ECCl e com a EAPCl, o que mostra a relação existente entre a satisfação acadêmica com a comunicação interpessoal do enfermeiro, ou seja, quanto maior a satisfação acadêmica, melhor a habilidade em comunicação. A correlação positiva e significativa entre as escalas de comunicação, a ECCl e a EAPCl, indica a coerência entre os instrumentos (Figura 3).

Pela análise de regressão normal múltipla, para o escore total da $\mathrm{ECCl}$, a cada 10 pontos da ESEA, o escore da ECCl aumentou, em média, 0,75 ponto. A EAPCl, pela análise de regressão binomial negativa, a cada 1 aumento do escore do ESEA, aumentou, em média, $0,64 \%$ o escore da EAPCI.

Figura 3 - Correlações estatisticamente significativas entre as escalas. ESEA versus ECCI; ESEA versus EAPCI; EAPCI versus ECCI. Guarulhos, SP, Brasil, 2016. (N=397)
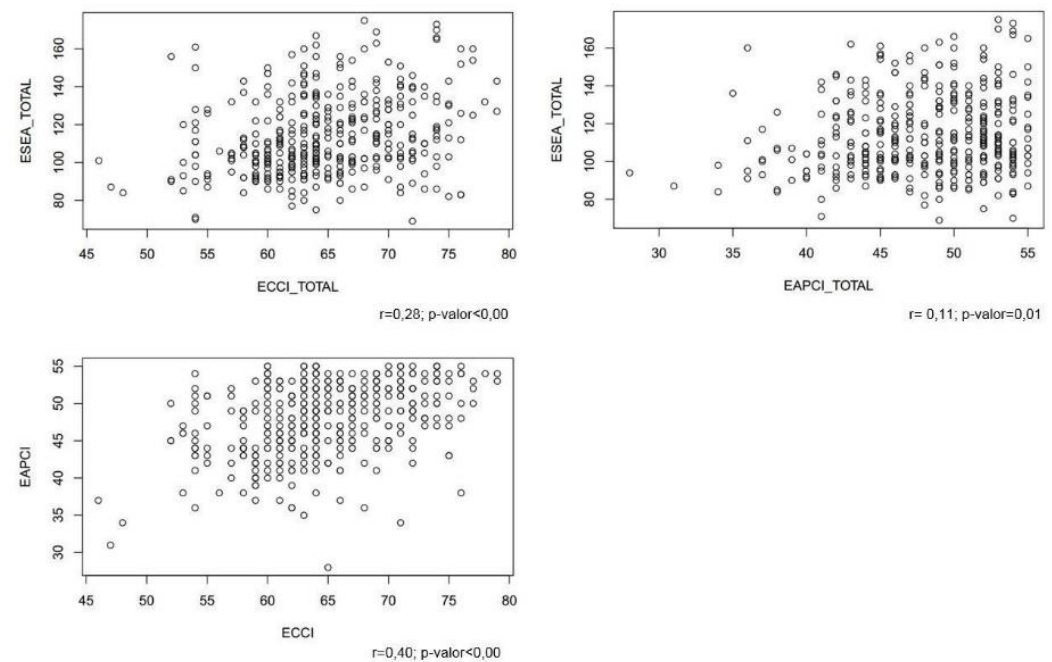

Fonte: Elaboração Própria (2020).

Legenda: ESEA: Escala de Satisfação com a Experiência Acadêmica de Estudantes do Ensino Superior. ECCI: Escala de Competência em Comunicação Interpessoal. EAPCI: Escala de Autoavaliação sobre Profissionalismo e Competência em Comunicação Interpessoal entre Enfermeiros e Pacientes. r: razão de Correlação de Spearman. p-valor: nível de significância.

Referente às variáveis numéricas com os domínios da ECCl, houve correlações significativas e negativas no domínio Disponibilidade com tempo de formação $(r=-0,10 ; p$-valor=0,03) e idade $(r=-$ 
$0,18 ; \quad p$-valor $=0,00)$, o que demonstra que enfermeiros com menor tempo de formação e mais jovens apresentam mais disponibilidade nas relações, expressando preocupação com as pessoas, permitindo que os outros os vejam como eles realmente são e olhando nos olhos dos indivíduos quando falam com eles. Não houve diferenças estatisticamente significativas entre as variáveis numéricas com a EAPCl.

Nos resultados da associação das características sociodemográficas, de formação e profissional com os domínios da $\mathrm{ECCl}$, houve diferenças estatisticamente significativas na comparação do domínio Autorrevelação com a instituição de formação, o que indica que enfermeiros que estudaram em instituições públicas têm maior habilidade para demonstrar sentimentos, ideias e pensamentos por meio da comunicação (Figura 4).

Associação estatisticamente significativa do domínio Manejo das Interações com formação prévia, na área de enfermagem, indica que aqueles que não possuíam formação prévia, como auxiliar de enfermagem, demonstram maior capacidade tanto de demonstrar compreensão quanto de perceber pelos sinais não verbais o que as pessoas sentem, principalmente, quando comparados com os auxiliares de enfermagem (Figura 4).

Houve também associação significativa do domínio Disponibilidade com a religião, em que os católicos se mostraram mais acessíveis, abertos e disponíveis tanto em relação aos evangélicos como aos espíritas (Figura 4).

Figura 4 - Associações estatisticamente significativas das variáveis sociodemográficas e profissionais com a ECCI. Guarulhos, SP, Brasil, 2016. ( $N=397)$

A
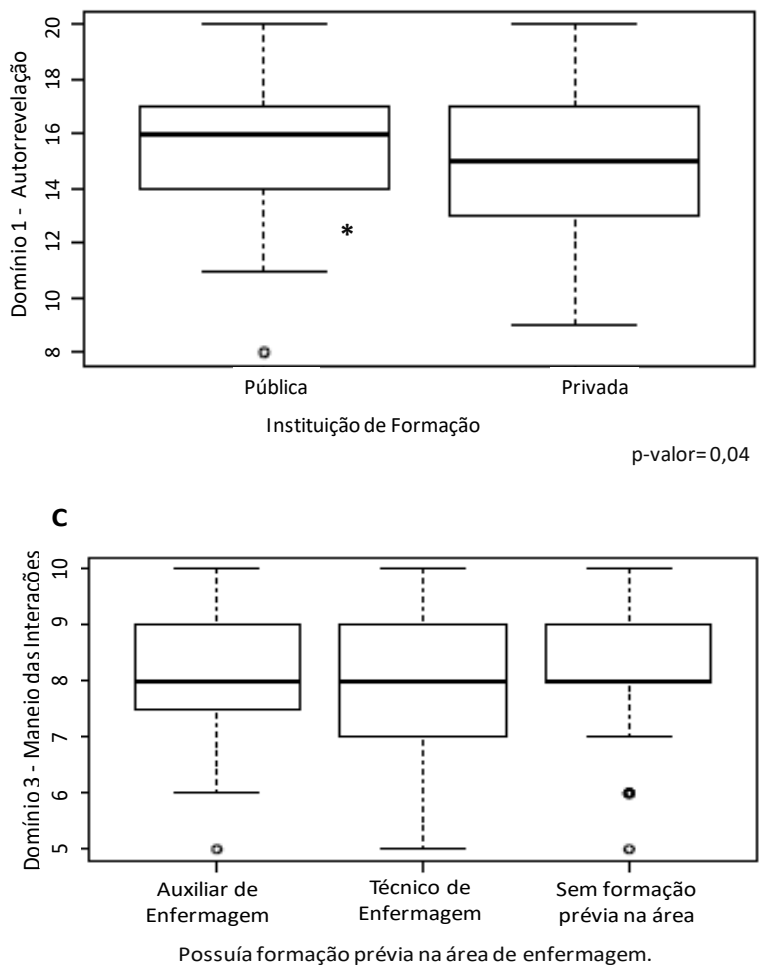

p-valor $=0,01$
B

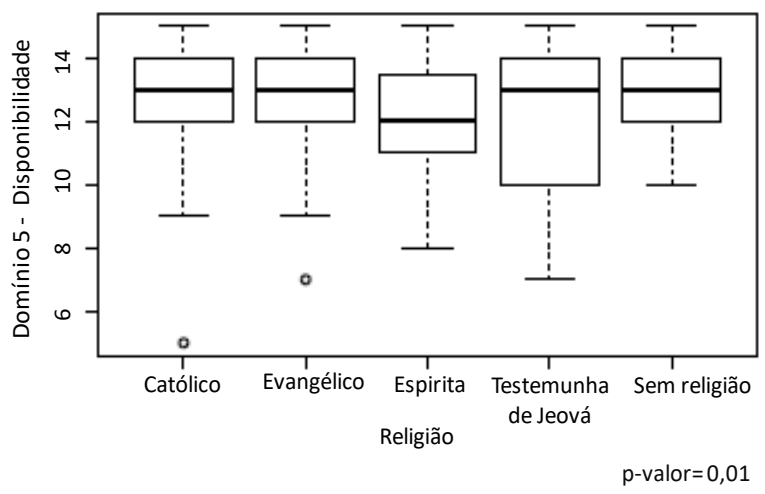

Fonte: Elaboração Própria (2020).

Legenda: ECCl: Escala de Competência em Comunicação Interpessoal. p-valor: nível de significância. Resultado da comparação dois a dois pelo método de Bonferroni: B: católica versus evangélica ( $p$-valor 0,04); católica versus espírita ( $p$-valor 0,00 ). C: sem formação prévia na área versus auxiliar de enfermagem ( $p$-valor 0,00). A: Teste Mann-Whitney. B e C: Teste Kruskal-Wallis.

Considerando a EAPCl, houve associações estatisticamente significativas no fator profissionalismo com sexo e com turno de trabalho, em que enfermeiros do sexo feminino e que atuam no turno integral classificam mais positivamente o seu profissionalismo que enfermeiros que atuam no período diurno ou noturno (Figura 5).

Foi também significativa a comparação do fator habilidade interpessoal com o turno de trabalho, o que mostra que enfermeiros que atuam no turno da manhã possuem maior capacidade em reagir da melhor forma à presença do outro, 
incluindo gestos utilizados, tratamento à outra pessoa, escuta e interesse pelo outro.

A comparação estatisticamente significativa do fator sinceridade na relação com religião mostra que tanto aqueles que se declararam católicos quanto sem religião declararam ter mais sinceridade nas relações enfermeiro-paciente e falam ao paciente o que é pertinente a ele saber durante o acompanhamento de sua saúde (Figura 5).

O fator troca de informação com turno de trabalho obteve associação estaticamente significativa e revela que profissionais que atuam no turno da manhã ou integral têm maior capacidade em transmitir e receber informações pertinentes, durante o exame físico, que aqueles que trabalham no período noturno (Figura 5).

Figura 5 - Associações estatisticamente significativas das variáveis sociodemográficas e profissionais com a EAPCI. Guarulhos, SP, Brasil, 2016. ( $\mathrm{N}=397)$
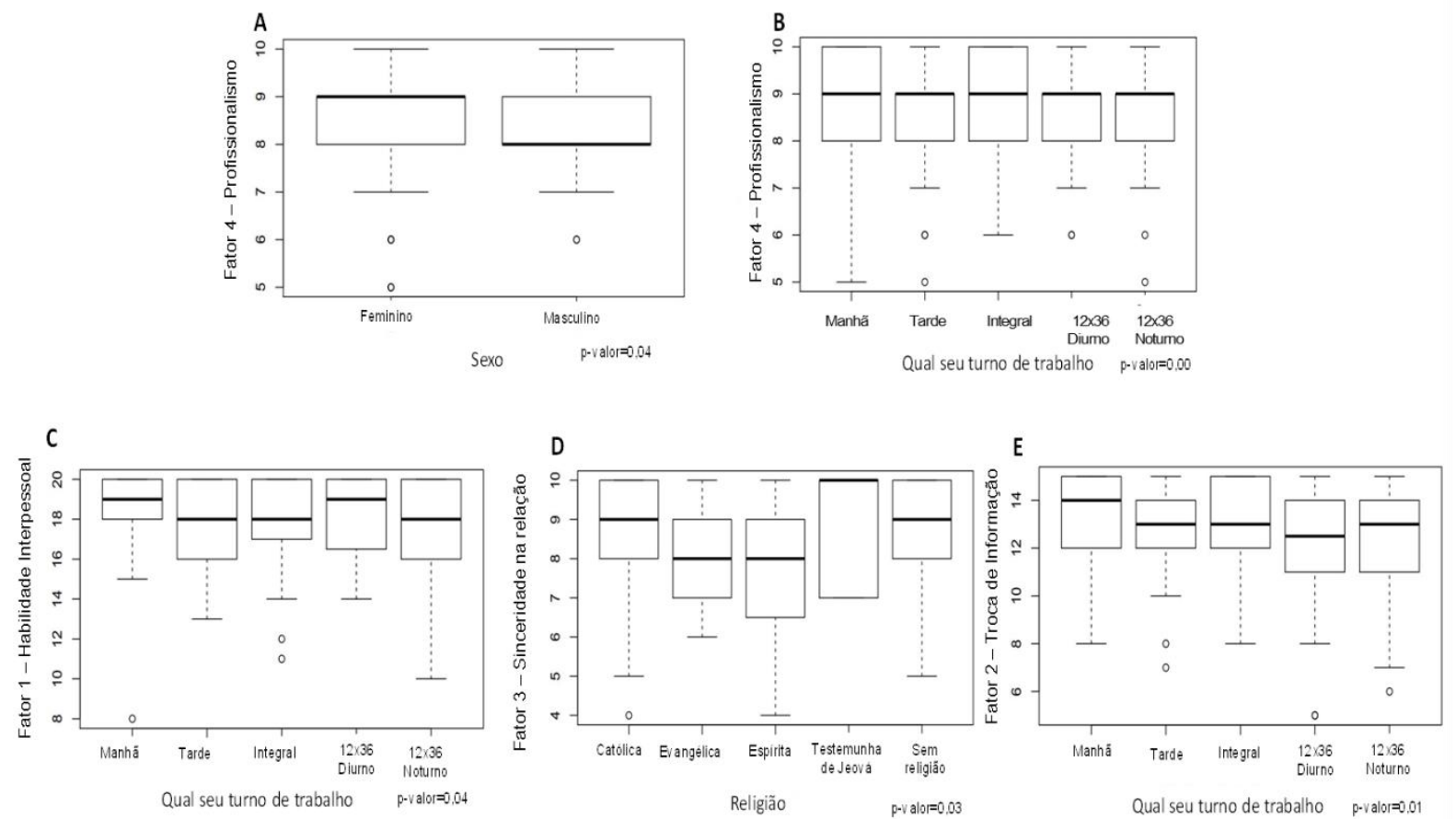

Fonte: Elaboração Própria (2020).

Legenda: EAPCl: Escala de Autoavaliação sobre Profissionalismo e Competência em Comunicação Interpessoal entre Enfermeiros e Pacientes. p-valor: nível de significância. Resultado da comparação dois a dois pelo método de Bonferroni: B: católica versus espirita ( $p$-valor 0,00 ); sem religião versus espirita ( $p$-valor 0,02$)$. C: manhã versus tarde ( $p$-valor 0,00 ), manhã versus $12 \times 36$ noturno ( $p$-valor 0,02 ). D: manhã versus $12 \times 36$ noturno ( $p$-valor 0,01 ), integral versus $12 \times 36$ noturno $(0,01)$. E: integral versus $12 \times 36$ diurno ( $p$-valor 0,00 ), integral versus $12 \times 36$ noturno ( $p$-valor 0,00). A: Teste Mann-Whitney. B, C, D e E: Teste Kruskal-Wallis.

Neste estudo, os enfermeiros se consideram, em geral, satisfeitos com a experiência acadêmica. Além disso, a satisfação acadêmica aumenta com a competência em comunicação interpessoal e melhor autoavaliação do profissionalismo. Esses achados corroboram com os encontrados em outros estudos da literatura(12-15).

A satisfação acadêmica é mais alta, quando os estudantes de enfermagem se sentem incluídos, apoiados durante sua jornada de aprendizado, têm oportunidade de envolvimento com colegas e funcionários da instituição de ensino, ou seja, possuem memórias positivas das relações interpessoais nesse período de formação ${ }^{(12)}$.
Estudantes que realizavam atividades extracurriculares percebiam-se mais satisfeitos com a instituição, por sentirem-se motivados, melhor preparados para o mercado de trabalho e terem maior desempenho na aprendizagem ${ }^{(16)}$. Essas atividades extracurriculares certamente proporcionaram outras experiências e oportunidades relacionais para o aluno.

Em outro estudo, destaca-se a importância de o aluno vivenciar situações da área de atuação. A formação deve proporcionar-lhe o conhecimento necessário, para adquirir habilidades técnico-científicas e habilidades relacionais, sensibilizando-o e preparando-o para enfrentar as situações no ambiente de trabalho ${ }^{(15)}$. 
A relação satisfação acadêmica e comunicação se dá provavelmente pelas características da profissão e funções do enfermeiro. A habilidade em comunicação interpessoal é um elemento básico para o cuidar. O cuidado necessita tanto do conhecimento técnico-prático, como também do envolvimento com o outro e o uso das habilidades comunicacionais.

Deve-se buscar de modo permanente o aperfeiçoamento do conhecimento e das habilidades em relações interpessoais, pois a sua falta pode distanciar o enfermeiro da assistência direta ao paciente e, além disso, tornar essas relações superficiais, impessoais e distanciadas do cuidado ideal(17). A capacitação do enfermeiro, para a utilização das habilidades interpessoais, potencializa as possibilidades de relacionamentos terapêuticos e a superação de barreiras nas relações com o paciente, família e equipe ${ }^{(18)}$.

Espera-se que a formação prepare e contemple conteúdos, para o desenvolvimento de competências e habilidades, trazendo confiança para o enfermeiro executar o que foi aprendido, em seu ambiente de trabalho, diante da complexidade das relações e dos cuidados de enfermagem ${ }^{(19)}$.

$\mathrm{Na}$ atual pesquisa, a disponibilidade para a comunicação, perceber e compreender o outro foi maior nos enfermeiros mais jovens e com menor tempo de formação. Com o decorrer do tempo, os valores pessoais sofrem variações. No início de carreira, há maior tendência à abertura a mudanças, disposição para a exploração, valorização e estimulação, indicando que, ao longo da trajetória profissional, os valores e atitudes sofrem variações em decorrência das prioridades no trabalho ${ }^{(20)}$.

O achado de que a interação com o outro que envolve o feedback bidirecional, do envolvimento foi maior nos enfermeiros que não possuíam formação prévia na área de enfermagem, não eram auxiliares nem técnicos de enfermagem, converge para a questão da idade e tempo de formação, pois, sem experiência prévia, o prazer da descoberta é notório e faz com que o indivíduo preste mais atenção às suas ações e reações.

Um estudo realizado no Irá identificou uma relação significativa entre idade e a comunicação de enfermeiras que atuavam em centros de saúde. A capacidade de fala, escuta e a frequência de feedbacks diminuíram com o aumento da idade, evidenciando uma redução na disposição para a comunicação com o passar do tempo(21).

Outra evidência encontrada neste estudo foi que o tipo de instituição, pública ou privada, influenciou na comunicação interpessoal; os que estudaram em universidades públicas mostraram mais habilidade para expressar sentimentos e pensamentos.

Os achados de uma pesquisa realizada com enfermeiros egressos de uma faculdade pública de Istambul, Turquia, corroboram com os atualmente encontrados. Os profissionais formados em instituição pública se diferenciavam dos demais profissionais, possuíam um desempenho melhor no conhecimento, expectativas profissionais, trabalho em equipe e na comunicação( ${ }^{(22)}$.

Neste estudo, o profissionalismo foi mais bem classificado em participantes do sexo feminino e que trabalham no turno integral. Trabalhar a autoavaliação do profissional permite avaliar o próprio desempenho e reconhecer os pontos fortes e o que ainda precisa ser melhorado. É importante que o profissional analise crítica e constantemente o próprio trabalho ${ }^{(23)}$.

Os estudantes do sexo feminino exibem maior empatia que os estudantes do sexo masculino. A empatia é um importante elemento do cuidar, pois fortalece a relação entre paciente e profissional de saúde, melhora a satisfação profissional e, consequentemente, promove melhores resultados clínicos para o paciente ${ }^{(24)}$.

A principal limitação deste estudo foi não ter sido possível avaliar a dimensão da comunicação não verbal dos enfermeiros. As escalas utilizadas enfocam a comunicação verbal, sendo pertinentes outros estudos que avaliem a comunicação em outras dimensões.

\section{CONCLUSÕES}

Quanto mais satisfeitos com a experiência acadêmica, maior a competência em comunicação interpessoal e melhor é a autoavaliação sobre seu profissionalismo.

A comunicação interpessoal difere de forma positiva com o tempo de formação, idade, sexo e religião, bem como com o tipo da instituição de formação, ter ou não formação prévia na área de enfermagem e turno de trabalho. A percepção do indivíduo sobre o seu profissionalismo modifica-se com o sexo e o turno de trabalho.

Este estudo também contribuiu para que os enfermeiros tenham a oportunidade de refletir sobre a relação entre o conhecimento e formação 
com as habilidades de comunicação, sendo assim, pode-se afirmar que o aprimoramento das interações do enfermeiro com o paciente, família e equipe, dependem tanto do conhecimento quanto da habilidade comunicacional.

\section{REFERÊNCIAS}

1- Porto MAS, Soares AB. Diferenças entre expectativas e adaptação acadêmica de universitários de diversas áreas do conhecimento. Anál Psicol. 2017;31(1):13-24. DOI: 10.14417/ap.1170

2- Carvalho G, Lopes S. Satisfação profissional do enfermeiro em uma unidade de emergência de hospital geral. Arq Ciênc Saúde 2006 [citado em 5 abr 2018]; 13(4):215-9. Available from: http://repositorio-racs.famerp.br/racs ol/vol-134/Famerp\%2013(4)\%20ID\%20210\%20-\%2017.pdf

3- Brasil. Ministério da Educação e Cultura. Conselho Nacional de Educação. Resolução CNE/CES n ${ }^{\circ} 3$, de 7 de novembro de 2001. Institui Diretrizes curriculares do curso de graduação em enfermagem. Diário Oficial da União 2001.

4- Vaismoradi M, Salsali M, Ahmadi F. Perspectives of Iranian male nursing students regarding the role of nursing education in developing a professional identity: a content analysis study. Jpn J Nurs Science 2011;8(2):174-83. DOI: $\underline{10.1590 / 010407072015002870013}$

5- Backers DS, Erdmann AL. Formação do enfermeiro pelo olhar do empreendedorismo social. Rev Gaúcha Enferm. 2009 [citado em 23 jun 2017]; 30(2);242-8. Available from: http://www.seer.ufrgs.br/index.php/RevistaGauch adeEnfermagem/article/view/7252/6681

6- Camelo SHH. Competência profissional do enfermeiro para atuar em Unidades de Terapia Intensiva: uma revisão integrativa. Rev Latino-Am Enfermagem 2012;20(1):1-9. DOI: 10.1590/S0104$\underline{11692012000100025}$

7- Esperidião E, Munari DB. Holismo só na teoria: a trama de sentimentos do acadêmico de enfermagem sobre sua formação. Rev Esc Enferm USP 2004;38(3):332-40. DOI: 10.1590/50080$\underline{62342004000300012}$

8- Faria MFG, Fernandes SG, Pirolo SM, Silva MJP. Falar em público: visão do mestrando de enfermagem. Rev Esc Enferm USP 1998;32(1):5866. DOI: $10.1590 /$ S0080-62341998000100009

9- Silva MJP. Comunicação tem remédio. A comunicação nas relações interpessoais em saúde. 10a ed. São Paulo: Edições Loyola; 2015.

10- Santos AAA, Polydoro SOJ, Scortegagna SA, Linden MAS. Integração ao ensino superior e satisfação acadêmica em universitários. Psicol Ciênc Prof. 2013;33(4):780-93. DOI: 10.1590/010407072015002870013

11- Schleich ALG, Polydoro SAJ, Santos AAA. Escala de satisfação com a experiência acadêmica de estudantes do ensino superior. Aval Psicol. 2006 [citado em 6 dez 2015]; 5(1):11-20. Available from: http://pepsic.bvsalud.org/pdf/avp/v5n1/v5n1a03. pdf.

12- Johnson $M$, Cowin LS, Wilson I, Young $H$. Professional identity and nursing: contemporany theoretical developments and future research challenges. Int Nurs Rev. 2012;59(4):562-9. DOI: 10.1111/j.1466-7657.2012.01013.x/abstract

13- Pereira TJ, Puggina AC. Validação do selfassesment of communication skills and professionalism para enfermeiros. Rev Bras Enferm. 2017;70(3):616-22. DOI: 10.1590/00347167-2016-0133

14- Puggina AC, Silva MJP. Interpersonal Communication Competence Scale: brazilian translation, validation and cultural adaptation. Acta Paul Enferm. 2014;27(2):108-14. DOI: 10.1590/1982-0194201400020

15- Vieira MA, Souto LES, Souza SM, Lima CA, Ohara CVS, Domenico EBL. Diretrizes curriculares nacionais para a área da enfermagem: o papel das competências na formação do enfermeiro. Rev Norte Min Enferm. 2016 [citado em 23 jun 2017]; 5(1):105-21. Available from: http://www.renome.unimontes.br/index.php/ren ome/article/view/102/148

16- Ramos MR, Barlem JGT, Lunardi VL, Barlem ELD, Silveira RS, Bordignon SS. Satisfaction with academic experience among undergraduate nursing students. Texto Contexto Enferm. 2015;24(1):187-95. DOI: 10.1590/0104$\underline{07072015002870013}$

17- Formozol GA, Oliveira DC, Costa TL, Gomes AMT. As relações interpessoais em saúde: uma 
aproximação ao problema. Rev Enferm UERJ 2012 [citado em 20 jan 2018]; 20(1):124-7. Available from: $\quad$ http://www.facenf.uerj.br/v20n1/ $\underline{\text { v20n1a21.pdf }}$

18- Bhana VM. Interpersonal skills development in generation y student nurses: a literature review. Nurse Educ Today 2014;34(12): 1430-4. DOI: 10.1016/i.nedt.2014.05.002

19- Costa RRO, Medeiros SM, Coutinho VRD, Mazzo A, Araújo MS. Satisfaction and selfconfidence in the learning of nursing students: randomized clinical trial. Esc Anna Nery 2019;24(1):e20190094. DOI: 10.1590/2177-9465ean-2019-0094

20- Reis GC. Os valores dos administradores de empresas mudam ao longo da carreira? Relações entre prioridades axiológicas e tempo de formado. Rev Adm Mackenzi 2010;11(5):32-54. DOI: $\underline{10.1590 / \text { S1678-69712010000500003 }}$

21- Hasan SH, Nesami MB, Nia RD, Nezhad FR, Akbari $\mathrm{H}$, Balaghafari A, Vahdei ME. Assessment of Interpersonal Communication Skills Among Sari Health Centers' Staff. Mater Sociomed. 2014 [citado 05 abr 2018] 26(5):324-328. Available from: DOI: $105455 / \mathrm{msm} .2014 .26 .324-328$

22- Altuntas S, Baykal, U. An analysis alumni performance: a study of the quality of nursing education. Nurse Educ Today 2016;49:135-9. DOI: $\underline{0.1016 / \text { i.nedt.2016.11.022 }}$

23- Ribeiro EAG, Punhagui GC, Souza NA. Autoavaliação $x$ autoanotação - aproximação e afastamentos na formação de professores autorregulados. Educação 2014;39(2):403-14. DOI: $\underline{10.5902 / 198464446379}$

24- Petrucci C, La Cerra C, Aloisio F, Montanari P, Lancia L. Empathy in health professional sudents: a comparative cross-sectional study. Nurse Educ Today 2016;41:1-5. DOI:

\subsection{6/j.nedt.2016.03.022}

Nota: Pesquisa Financiada pela Coordenação de Aperfeiçoamento de Pessoal de Nível Superior.

Artigo Extraído da Dissertação "Relação da satisfação acadêmica, características sociodemográficas e profissionais com a comunicação interpessoal do enfermeiro.

Recebido em: $23 / 12 / 20$

Aprovado em: 16/00/20 